\title{
A Pre-FFT OFDM Adaptive Array Antenna with Eigenvector Combining
}

\author{
Shinsuke HARA ${ }^{\dagger a}$, Member, Quoc Tuan TRAN ${ }^{\dagger \dagger}$, Yunjian JIA ${ }^{\dagger \dagger}$, Student Members, \\ Montree BUDSABATHON ${ }^{\dagger+\dagger}$, Nonmember, and Yoshitaka HARA ${ }^{+\dagger^{+\dagger}+}$, Member
}

\begin{abstract}
SUMMARY This paper proposes a novel pre-FFT type OFDM adaptive array antenna called "Eigenvector Combining." The eigenvector combining array antenna is a realization of a post-FFT type OFDM adaptive array antenna through a pre-FFT signal processing, so it can achieve excellent performance with less computational complexity and shorter training symbols. Numerical results demonstrate that the proposed eigenvector combining array antenna shows excellent bit error rate performance close to the lower bound just with 2 OFDM symbol-long training symbols.

key words: OFDM, adaptive array antenna, co-channel interference suppression, eigenvector
\end{abstract}

\section{Introduction}

Orthogonal frequency division multiplexing (OFDM), which is a pre-distortion or an equalization technique at transmit side in a sense, is an efficient technique for highspeed digital transmission over severe multipath fading channels [1], and recently has been considered to be a promising technique for next generation mobile communications systems [2]. OFDM inserts a cyclic prefix in every OFDM symbol so as to increase system robustness against delayed signals, however, once delayed signals beyond the cyclic prefix, namely, co-channel interfering signals, are introduced in a channel with larger delay spread, there is a severe degradation in the transmission performance.

To maintain high-speed reliable wireless communications systems, the use of multiple antennas at receive side has been considered as an effective tool not only for gain enhancement, increased spectral efficiency [3] but also for interference suppression [4]. Although a post-fast Fourier transform (FFT) subcarrier-by-subcarrier combining OFDM adaptive array antenna is optimum in terms of maximizing signal-to-interference-and-noise power ratio (SINR), it requires the increased number of FFT processors and heavy computations which increase with the number of antennas

\footnotetext{
Manuscript received August 1, 2005.

Manuscript revised December 30, 2005.

${ }^{\dagger}$ The author is with the Graduate School of Engineering, Osaka City University, Osaka-shi, 558-8585 Japan.

${ }^{\dagger}$ The author is with the Graduate School of Engineering, Osaka University, Suita-shi, 565-0871 Japan.

${ }^{\dagger \dagger}$ The author is with Hitachi Central Research Laboratory, Kokubunji-shi, 185-8601 Japan.

${ }^{\dagger+\dagger}$ The author is with Fabrinet Co. Ltd., Patumthanee, Thailand.

${ }^{+1+\dagger \dagger}$ The author is with Mitsubishi Electric Information Technology Centre Europe B.V. (ITE), Rennes, France.

a)E-mail: hara@comm.eng.osaka-u.ac.jp

DOI: 10.1093/ietcom/e89-b.8.2180
}

and subcarriers, and furthermore it requires quite long training symbols [5]. Related to the post-FFT configuration, a direction of arrival (DoA)-based technique is proposed for co-channel interference suppression in [6], and a minimum mean square error (MMSE)-based technique is proposed for space diversity in [7]. On the other hand, a pre-FFT type OFDM adaptive array antenna, which requires only one FFT processor, can drastically reduce the computational complexity by tolerating some performance degradation [8], [9].

This paper proposes a novel pre-FFT type OFDM adaptive array antenna called "Eigenvector Combining [10]." In [11], the performance of the eigenvector combining OFDM adaptive array antenna is discussed as a gain enhancement/interference suppression tool for the case where there are desired and co-channel interfering signals. Almost at the same time, in [12], the same OFDM adaptive array antenna is proposed as a space diversity tool. If there is no interference, the optimal post-FFT type OFDM adaptive array antenna has the structure of a maximal ratio subcarrierby-subcarrier combining diversity, so its realization through pre-FFT signal processing is not so advantageous. Discussions for co-channel interference suppression is necessary, because the eigenvector combining has the characteristics of the two types of the array antennas, namely, excellent performance inherent in the post-FFT type and less computational complexity and requirement of shorter training symbols proper to the pre-FFT type.

The paper is organized as follows. Section 2 shows the general system model. Section 3 shows the structures of the post-FFT and pre-FFT type OFDM adaptive array antennas. Section 4 presents the principle of the eigenvector combining array antenna. Section 5 shows a simple theoretical proof on the equivalence between the post-FFT type and eigenvector combining array antennas. Section 6 compares the computational complexity among the post-FFT, pre-FFT and eigenvector combining array antennas. Section 7 shows the computer simulation results and discusses the performance of the three array antennas. Finally, Sect. 8 presents some conclusions.

\section{System Model}

Figure 1(a) shows the block diagram of an OFDM transmitter. The data sequence is first convolutionally encoded, then the encoded bit is mapped onto a quadrature phase shift keying (QPSK) symbol after bit-level interleaving. The QPSK 


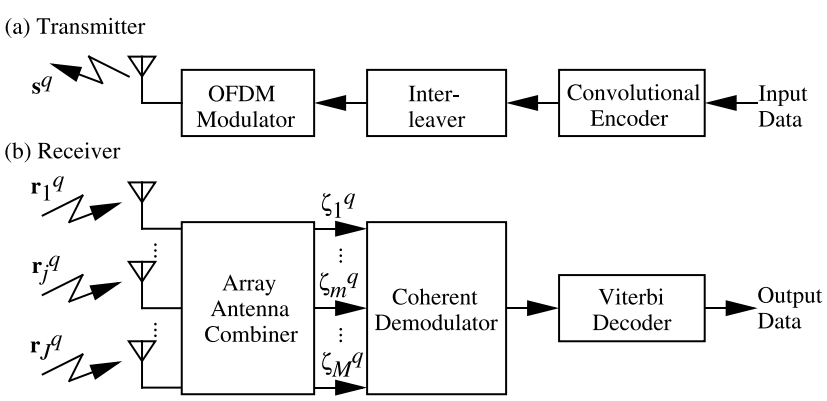

Fig. 1 System model: OFDM transmitter (a) and OFDM receiver (b).

symbols are mapped onto one OFDM symbol through the inverse FFT device, and after a cyclic prefix is inserted in each OFDM symbol, the cyclically prefixed OFDM symbols are finally transmitted.

Defining the lengths of the cyclic prefix and useful symbol as $L$ and $M$, respectively, the $q$ th OFDM symbol vector $((M+L) \times 1)$ in the equivalent baseband expression is written as

$$
\begin{aligned}
\mathbf{s}^{q} & =\left[s_{1}^{q}, \cdots, s_{M+L}^{q}\right]^{T} \\
& =\mathbf{G}_{i n s} \mathbf{F}^{H} \boldsymbol{\alpha}^{q} .
\end{aligned}
$$

In (1), $T$ and $H$ denote transposition and Hermitian transposition, respectively, and $\mathbf{G}_{\text {ins }}, \mathbf{F}$ and $\boldsymbol{\alpha}^{q}$ are the cyclic prefix insertion matrix $((M+L) \times M)$, the $M$-point discrete Fourier transform (DFT) matrix $(M \times M)\left(\mathbf{F}^{H}\right.$ denotes the $M$-point inverse DFT matrix) and the $q$ th data symbol vector transmitted over $M$ subcarriers $(M \times 1)$, respectively, which are given by

$$
\begin{aligned}
& \mathbf{G}_{i n s}= {\left[\begin{array}{c}
\mathbf{0}_{L \times(M-L)}, \mathbf{I}_{L \times L} \\
\mathbf{I}_{M \times M}
\end{array}\right] } \\
& \mathbf{F}=\left\{f_{t, u}\right\} \\
& f_{t, u}=\frac{1}{\sqrt{M}} e^{-j 2 \pi \frac{(t-1)(u-1)}{M}} \\
& \quad(t, u=1, \cdots, m, \cdots, M) \\
& \boldsymbol{\alpha}^{q}=\left[\alpha_{1}^{q}, \cdots, \alpha_{m}^{q}, \cdots, \alpha_{M}^{q}\right]^{T}
\end{aligned}
$$

where $\mathbf{0}_{a \times b}$ and $\mathbf{I}_{a \times a}$ denote the matrix with size of $a \times b$ whose elements are all 0 s and the identity matrix with size of $a \times a$, respectively.

Through a channel, the transmitted signal is subject to frequency selective Rayleigh fading and it is furthermore contaminated with interference and noise. Figure 1(b) shows the block diagram of an OFDM receiver which is equipped with $J$ antenna elements. The received signals at all the array elements are appropriately processed, namely, the part of array antenna combiner is replaced by a post-FFT type, a pre-FFT-type and the eigenvector combining, which are shown in Figs. 2, 3 and 4, respectively. The Viterbi decoder finally gives the estimates for the transmitted data after coherent demodulation.

Defining the received wave vector $((M+L) \times 1)$ for the $q$ th OFDM symbol at the $j$ th antenna element as $\mathbf{r}_{j}^{\prime q}$, the received wave vector $(M \times 1)$ after removal of cyclic prefix is written as $(j=1, \cdots, J)$

$$
\begin{aligned}
\mathbf{r}_{j}^{q} & =\left[r_{j 1}^{q}, \cdots, r_{j m}^{q}, \cdots, r_{j M}^{q}\right]^{T} \\
& =\mathbf{G}_{r e m} \mathbf{r}_{j}^{q} \\
& =\mathbf{x}_{j}^{q}+\mathbf{y}_{j}+\mathbf{n}_{j}
\end{aligned}
$$

where $\mathbf{G}_{r e m}, \mathbf{x}_{j}^{q}, \mathbf{y}_{j}$ and $\mathbf{n}_{j}$ are the cyclic prefix removal matrix $(M \times(M+L))$, the $q$ th received OFDM signal vector $(M \times 1)$, the interference vector $(M \times 1)$ and the noise vector $(M \times 1)$, respectively, which are given by

$$
\begin{aligned}
& \mathbf{G}_{r e m}=\left[\mathbf{0}_{M \times L}, \mathbf{I}_{M \times M}\right] \\
& \mathbf{x}_{j}^{q}=\left[x_{j 1}^{q}, \cdots, x_{j m}^{q}, \cdots, x_{j M}^{q}\right]^{T} \\
& \mathbf{y}=\left[y_{j 1}, \cdots, y_{j m}, \cdots, y_{j M}\right]^{T} \\
& \mathbf{n}=\left[n_{j 1}, \cdots, n_{j m}, \cdots, n_{j M}\right]^{T} .
\end{aligned}
$$

Here assume that these vectors satisfy the following properties:

$$
\begin{aligned}
& E\left[\mathbf{x}_{j}^{q} \mathbf{y}_{j}^{H}\right]=\mathbf{0}_{M \times M} \\
& E\left[\mathbf{x}_{j}^{q} \mathbf{n}_{j}^{H}\right]=\mathbf{0}_{M \times M} \\
& E\left[\mathbf{y}_{j} \mathbf{n}_{j}^{H}\right]=\mathbf{0}_{M \times M} \\
& E\left[\mathbf{n}_{j} \mathbf{n}_{j}^{H}\right]=\sigma_{n}^{2} \mathbf{I}_{M \times M}
\end{aligned}
$$

where $E[\cdot]$ denotes the ensemble average and $\sigma_{n}^{2}$ denotes the power of noise.

Before going to the detail analyses on the post-FFT type, pre-FFT type and eigenvector combining OFDM adaptive array antennas, define the received wave matrix $(J \times M)$ as

$$
\begin{aligned}
\mathbf{R}^{q} & =\left[\mathbf{r}_{1}^{q}, \cdots, \mathbf{r}_{j}^{q}, \cdots, \mathbf{r}_{J}^{q}\right]^{T} \\
& =\mathbf{X}^{q}+\mathbf{Y}+\mathbf{N} \\
\mathbf{X}^{q} & =\left[\mathbf{x}_{1}^{q}, \cdots, \mathbf{x}_{j}^{q}, \cdots, \mathbf{x}_{J}^{q}\right]^{T} \\
\mathbf{Y} & =\left[\mathbf{y}_{1}, \cdots, \mathbf{y}_{j}, \cdots, \mathbf{y}_{J}\right]^{T} \\
\mathbf{N} & =\left[\mathbf{n}_{1}, \cdots, \mathbf{n}_{j}, \cdots, \mathbf{n}_{J}\right]^{T} .
\end{aligned}
$$

Finally, defining the channel impulse response vector $(M \times 1)$ at the $j$ th antenna element as

$$
\mathbf{h}_{j}=\left[h_{j 1}, \cdots, h_{j m}, \cdots, h_{j M}\right]^{T}
$$

$\mathbf{X}^{q}$ can be written as

$$
\begin{aligned}
& \mathbf{X}^{q}=\mathbf{H S}^{q} \\
& \mathbf{H}=\left[\mathbf{h}_{1}, \cdots, \mathbf{h}_{j}, \cdots, \mathbf{h}_{J}\right]^{T} \\
& \mathbf{S}^{q}=\left\{s_{t, u}\right\} \\
& s_{t, u}=\left\{\begin{array}{c}
s_{L+(2-t)+(u-1)}^{q}, \\
(L+(2-t)+(u-1)>0) \\
s_{(M+L)+L+(2-t)+(u-1)}^{q-1}, \\
(L+(2-t)+(u-1) \leq 0)
\end{array}\right.
\end{aligned}
$$

where $\mathbf{H}$ and $\mathbf{S}^{q}$ denote the channel impulse response matrix $(J \times M)$ and the OFDM symbol matrix $(M \times M)$, respectively. Note that the OFDM symbol has a noise-like nature with the 
following important property: [9]

$$
E\left[\mathbf{S}^{q} \mathbf{S}^{q H}\right]=M \sigma_{s}^{2} \mathbf{I}_{M \times M}
$$

where $\sigma_{s}^{2}=E\left[s_{m}^{q \text { or }}{ }^{-1} s_{m}^{*}{ }^{\text {q or } q-1}\right]$ denotes the power of OFDM symbol.

\section{OFDM Adaptive Array Antennas}

\subsection{Post-FFT Type OFDM Adaptive Array Antenna}

Figure 2 shows the block diagram of the post-FFT type OFDM adaptive array antenna. The FFT output vector for the $q$ th OFDM symbol at the $j$ th antenna element $(M \times 1)$ is written as

$$
\begin{aligned}
\boldsymbol{\rho}_{j}^{q} & =\left[\rho_{j 1}^{q}, \cdots, \rho_{j m}^{q}, \cdots, \rho_{j M}^{q}\right]^{T} \\
& =\mathbf{F r}_{j}^{q} .
\end{aligned}
$$

In (25), $\rho_{j m}^{q}$ is also written as

$$
\rho_{j m}^{q}=\mathbf{f}_{m}^{T} \mathbf{r}_{j}^{q}
$$

where $\mathbf{f}_{m}^{T}$ is the $m$ th row vector $(1 \times M)$ for $\mathbf{F}$

$$
\mathbf{f}_{m}=\left[1, e^{-j 2 \pi m / M}, \cdots, e^{-j 2 \pi m(M-1) / M}\right]^{T} .
$$

Defining the FFT output vector for the $m$ th subcarrier component $(J \times 1)$ as

$$
\begin{aligned}
\boldsymbol{\xi}_{m}^{q} & =\left[\rho_{1 m}^{q}, \cdots, \rho_{j m}^{q}, \cdots, \rho_{J m}^{q}\right]^{T} \\
& =\mathbf{R}^{q} \mathbf{f}_{m}
\end{aligned}
$$

the post-FFT combined array output for the $m$ th subcarrier is written as

$$
\begin{aligned}
\zeta_{m}^{q \text { post }} & =\mathbf{w}_{m}^{\text {post }{ }^{H}} \boldsymbol{\xi}_{m}^{q} \\
& =\mathbf{w}_{m}^{\text {post }{ }^{H}} \mathbf{R}^{q} \mathbf{f}_{m}
\end{aligned}
$$

where $\mathbf{w}_{m}^{\text {post }}$ denotes the array weight vector for the post-FFT type $(J \times 1)$ defined as

$$
\mathbf{w}_{m}^{\text {post }}=\left[w_{1 m}^{\text {post }}, \cdots, w_{j m}^{\text {post }}, \cdots, w_{J m}^{\text {post }}\right]^{T} .
$$

The optimal weight vector based on the MMSE criterion is given by a solution for the following minimization problem:

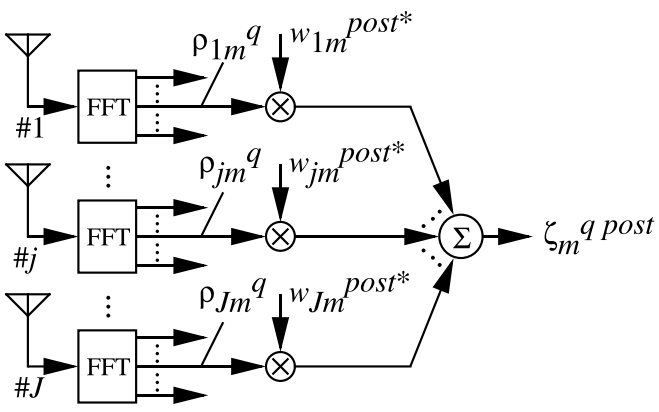

Fig. 2 Post-FFT type OFDM adaptive array antenna.

$$
\text { minimize } E\left[\left[\tilde{a}_{m}^{q}-\left.\mathbf{w}_{m}^{\text {post }}{ }^{H} \boldsymbol{\xi}_{m}^{q}\right|^{2}\right]\right.
$$

where $\widetilde{a}_{m}^{q}$ denotes the $q$ th known training symbol at the $m$ th subcarrier component. If the received signal contains no interference, the maximal ratio combining (MRC) is intuitively optimal [13], so

$$
\mathbf{w}_{m}^{p o s t}=\mathbf{H f}_{m} .
$$

In this case, few OFDM symbols are required to calculate the optimal weight vector as well as the channel impulse response matrix. On the other hand, if the received signal contains interference, direct evaluation of (31) is required to give the optimal solution. The minimization problem is solvable with an adaptive algorithm, however, conventional adaptive algorithms such as recursive least square (RLS) usually require several tens of symbols for good convergence [14]. It means that the post-FFT type OFDM adaptive array antenna requires several tens of known training OFDM symbols to calculate the weight vector.

\subsection{Pre-FFT Type OFDM Adaptive Array Antenna}

Figure 3 shows the block diagram of the pre-FFT type OFDM adaptive array antenna. The FFT input vector $(M \times 1)$ is written as

$$
\begin{aligned}
\mathbf{y}^{q T} & =\left[y_{1}^{q}, \cdots, y_{m}^{q}, \cdots, y_{M}^{q}\right] \\
& =\mathbf{w}^{\text {pre } H} \mathbf{R}^{q}
\end{aligned}
$$

where $\mathbf{w}^{p r e}$ is the array weight vector for the pre-FFT type $(J \times 1)$ defined as

$$
\mathbf{w}^{\text {pre }}=\left[w_{1}^{\text {pre }}, \cdots, w_{j}^{\text {pre }}, \cdots, w_{J}^{\text {pre }}\right]^{T} .
$$

The pre-FFT combined array output for the $m$ th subcarrier is written as

$$
\begin{aligned}
\zeta_{m}^{q \text { pre }} & =\mathbf{y}^{q T} \mathbf{f}_{m} \\
& =\mathbf{w}^{\text {pre }}{ }^{H} \mathbf{R}^{q} \mathbf{f}_{m} .
\end{aligned}
$$

Defining the powers of signal, interference and noise as $S, I$ and $N$, respectively, $S /(S+I+N)$ is given by

$$
\frac{S}{S+I+N}=\frac{\mathbf{w}^{\text {pre }{ }^{H}} \mathbf{C}_{x}^{q} \mathbf{w}^{\text {pre }}}{\mathbf{w}^{\text {pre } H} \mathbf{C}_{\text {rec }}^{q} \mathbf{w}^{\text {pre }}}
$$

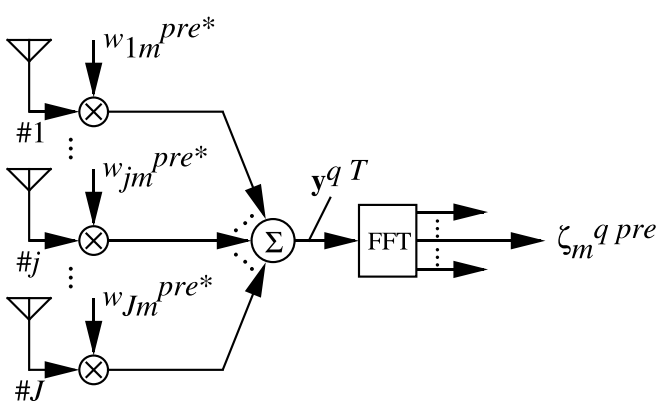

Fig. 3 Pre-FFT type OFDM adaptive array antenna. 
where $\mathbf{C}_{r e c}^{q}$ and $\mathbf{C}_{x}^{q}$ are the correlation matrices for the $q$ th received wave matrix and $q$ th received signal matrix, respectively, which are written as

$$
\begin{aligned}
& \mathbf{C}_{r e c}^{q}=E\left[\mathbf{R}^{q} \mathbf{R}^{q H}\right] \\
& \mathbf{C}_{x}^{q}=E\left[\mathbf{X}^{q} \mathbf{X}^{q H}\right]=\mathbf{H} E\left[\mathbf{S}^{q} \mathbf{S}^{q H}\right] \mathbf{H}^{H} .
\end{aligned}
$$

With (24), (38) can be written as

$$
\mathbf{C}_{x}^{q}=M \sigma_{s}^{2} \mathbf{H H}^{H} .
$$

On the other hand, with $\operatorname{SINR}, S /(S+I+N)$ is written as

$$
\frac{S}{S+I+N}=\frac{1}{1+S I N R^{-1}}
$$

so the optimal weight vector to maximize $S /(S+I+N)$ also maximizes SINR.

The maximization problem of (36) leads to the following generalized eigenproblem [9]:

$$
\begin{aligned}
& \text { find } \mathbf{w}^{\text {pre }} \text { which satisfies } \\
& \mathbf{C}_{x}^{q} \mathbf{w}^{\text {pre }}=\lambda_{\max } \mathbf{C}_{r e c}^{q} \mathbf{w}^{\text {pre }}
\end{aligned}
$$

where $\lambda_{\max }$ is the first largest eigenvalue [15]. When setting the array weight vector so as to satisfy (41), the largest SINR given by $\lambda_{\max }$ is realizable at the FFT input.

\section{Eigenvector Combining}

When the angular spread of received signals is small enough, the first largest generalized eigenvalue is dominant, so the pre-FFT type array antenna can utilize almost all part of the received signal power. In this case, there is no problem with the pre-FFT type array antenna. However, when the angular spread becomes larger, the other eigenvalues such as the second and third largest ones become larger (namely, their values become comparable with the value of the first largest one), so the pre-FFT type array antenna shows a power utilization inefficiency in the transmission performance. Utilization of the array weight vectors associated with the other larger eigenvalues can improve the transmission performance. This is the principle of the eigenvector combining.

(41) can be rewritten as

$$
\mathbf{C}_{x}^{q} \mathbf{w}(k)^{p r e}=\lambda(k) \mathbf{C}_{r e c}^{q} \mathbf{w}(k)^{\text {pre }}(k=1, \cdots, K)
$$

where there are assumed to be $K$ eigenvalues in the decreasing order for the signal subspace, namely, $\lambda(1)=\lambda_{\max } \geq$ $\lambda(2) \geq \cdots \lambda(K)$, and $\mathbf{w}(k)^{\text {pre }}$ is the array weight vector associated with the $k$ th eigenvalue $(J \times 1)$ defined as

$$
\mathbf{w}(k)^{\text {pre }}=\left[w(k)_{1}^{\text {pre }}, \cdots, w(k)_{j}^{\text {pre }}, \cdots, w(k)_{J}^{p r e}\right]^{T} .
$$

Figure 4(a) shows the structure of the proposed eigenvector combining OFDM adaptive array antenna through pre-FFT representation, which virtually has $K$ sets of preFFT type array antennas. The SINR of the $k$ th pre-FFT type array antenna is given by $\lambda(k)$, where the power of interference has been well suppressed. Therefore, the $K$ signals

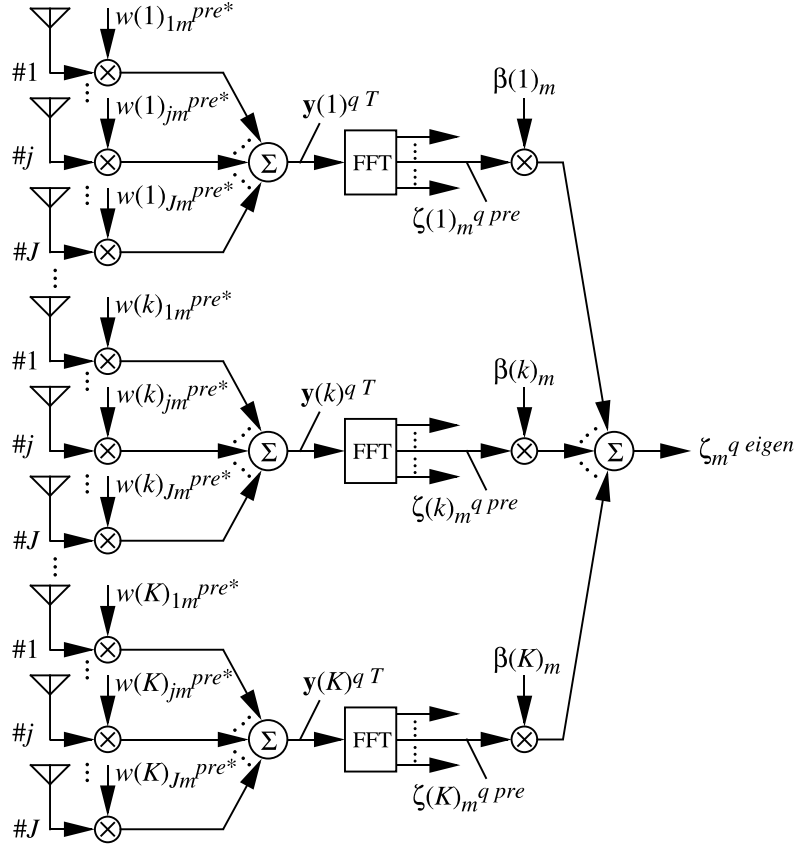

(a) Pre-FFT Representation

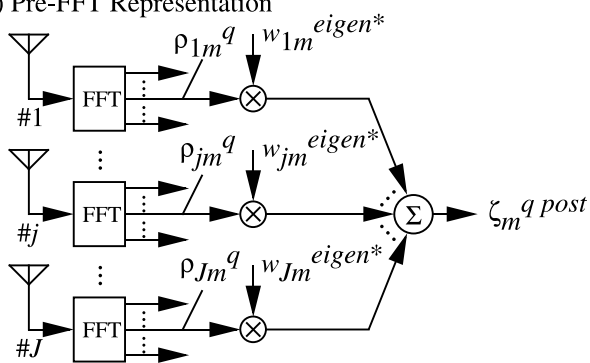

(b) Equivalent Post-FFT Representation

Fig. 4 Eigenvector combining: pre-FFT representation (a) and equivalent post-FFT representation (b).

after being combined with a set of $\mathbf{w}(k)^{\text {pre }}(k=1, \cdots, K)$ are coherently combined on subcarrier-by-subcarrier basis (also after FFT operation) in a maximum ratio combining (MRC) manner. Note that to perform the MRC properly, the following linear constraint needs to be imposed on Eq. (42):

$$
\text { subject to }\left|\mathbf{w}(k)^{\text {pre }}\right|=1 \text {. }
$$

With (33), the $q$ th FFT input vector at the $k$ th virtual branch $(M \times 1)$ is written as

$$
\begin{aligned}
\mathbf{y}(k)^{q T} & =\left[y(k)_{1}^{q}, \cdots, y(k)_{m}^{q}, \cdots, y(k)_{M}^{q}\right] \\
& =\mathbf{w}(k)^{\text {pre }} \mathbf{R}^{q}
\end{aligned}
$$

so the $m$ th subcarrier output at the $k$ th virtual array branch results in

$$
\begin{aligned}
\zeta(k)_{m}^{q \text { pre }} & =\mathbf{y}(k)^{q T} \mathbf{f}_{m} \\
& =\mathbf{w}(k)^{p r e ~}{ }^{\text {pr }} \mathbf{R}^{q} \mathbf{f}_{m} .
\end{aligned}
$$

On the other hand, the complex envelope for the $m$ th subcarrier component at the $k$ th virtual array branch is given by

$$
\beta(k)_{m}=\mathbf{w}(k)^{\text {pre } H} \mathbf{H f}_{m}
$$


therefore, the MRC-combined $m$ th final subcarrier output is written as

$$
\begin{aligned}
\zeta_{m}^{q \text { eigen }} & =\sum_{k=1}^{K} \beta(k)_{m}^{*} \zeta(k)_{m}^{q \text { pre }} \\
& =\left(\sum_{k=1}^{K} \beta(k)_{m}^{*} \mathbf{w}(k)^{\text {pre } H}\right) \mathbf{R}^{q} \mathbf{f}_{m} \\
& =\mathbf{f}_{m}^{H} \mathbf{H}^{H} \sum_{k=1}^{K}\left(\mathbf{w}(k)^{\text {pre }} \mathbf{w}(k)^{\text {pre } H}\right) \mathbf{R}^{q} \mathbf{f}_{m} .
\end{aligned}
$$

\section{Relationship between Eigenvector Combining and Post-FFT Type}

From (29) and (48), it can be seen that the eigenvector combining is a realization of a post-FFT type through pre-FFT signal processing as

$$
\zeta_{m}^{q \text { eigen }}=\mathbf{w}_{m}^{\text {eigen } H} \boldsymbol{\xi}_{m}^{q}
$$

where $\boldsymbol{\xi}_{m}^{q}$ is the the FFT output vector for the $m$ th subcarrier component $(J \times 1)$ defined by $(28)$ and $\mathbf{w}_{m}^{\text {eigen }}$ is the array weight vector for the eigenvector combining $(J \times 1)$ defined as

$$
\begin{aligned}
& \mathbf{w}_{m}^{\text {eigen }}=\left[w_{1 m}^{\text {eigen }}, \cdots, w_{j m}^{\text {eigen }}, \cdots, w_{J m}^{\text {eigen }}\right]^{T} \\
& w_{j m}^{\text {eigen }}=\sum_{k=1}^{K} \beta(k)_{m} w(k)_{j m}^{\text {pre }} .
\end{aligned}
$$

Therefore, the advantages of the eigenvector combining is clear, that is, it requires only few training OFDM symbols for array weight calculation due to pre-FFT processing and can utilize almost all part of received signal power. Figure 4(b) shows the post-FFT representation for the eigenvector combining.

For the case of no interference, the equivalence between the post FFT-type and eigenvector combining can be proved as follows. Taking the noise subspace into consideration, the summation term up to $J$ in (49) is simplified into

$$
\sum_{k=1}^{J}\left(\mathbf{w}(k)^{\text {pre }} \mathbf{w}(k)^{\text {pre } H}\right)=\mathbf{W}^{\text {pre }} \mathbf{W}^{\text {pre } H}
$$

where $\mathbf{W}^{\text {pre }}$ is the array weight matrix $(J \times J)$ defined as

$$
\mathbf{W}^{\text {pre }}=\left[\mathbf{w}(1)^{\text {pre }}, \cdots, \mathbf{w}(j)^{\text {pre }}, \cdots, \mathbf{w}(J)^{\text {pre }}\right]^{T} .
$$

When there is no interference, the generalized eigenvalue problem given by Eq. (41) becomes just an eigenvalue problem, where the eigenvectors are mutually orthogonal, so $\mathbf{W}^{\text {pre }}$ has the following property:

$$
\mathbf{W}^{\text {pre }{ }^{H}} \mathbf{W}^{\text {pre }}=\mathbf{I}_{J \times J} \text {. }
$$

$\mathbf{W}^{\text {pre }}$ has the full rank, so (55) means

$$
\mathbf{W}^{\text {pre } H}=\mathbf{W}^{\text {pre }-1} \text {. }
$$

Therefore, substituting (56) into (53) leads to

$$
\sum_{k=1}^{J}\left(\mathbf{w}(k)^{p r e} \mathbf{w}(k)^{\text {pre } H}\right)=\mathbf{I}_{J \times J}
$$

and finally, (49) becomes

$$
\zeta_{m}^{q \text { eigen }}=\left(\mathbf{H} \mathbf{f}_{m}\right)^{H} \mathbf{R}^{q} \mathbf{f}_{m}
$$

(58) shows the MRC-combined post-FFT type (see (29) and (32)).

\section{Computational Cost Comparison}

The number of FFT points, namely, the length of useful symbol, is often different from that of subcarriers in practical systems [16], so here we define the number of subcarriers as $M_{s u b}$. In addition, we define the number of training OFDM symbols required for the post-FFT type array antenna as $N_{\text {post }}$. In the following, the computational cost means the number or order $(O(\cdot))$ of complex multiplications.

The post-FFT type array antenna first needs to perform the FFT operations for $N_{\text {post }}$ training OFDM symbols to obtain all the subcarrier-level outputs at $J$ antenna elements. Taking into consideration the computational cost on the FFT operation is $O\left(M \log _{2} M\right)$ [17], the computational cost is $N_{\text {post }} \times J \times O\left(M \log _{2} M\right)$. Then, it needs to solve the minimization problem given by (31) to determine the array weight at each subcarrier. Assuming the sample matrix inversion (SMI) algorithm with computational cost of $O\left(J^{3}\right)$, it needs to calculate the correlation matrices $(J \times J)$ for $N_{\text {post }}$ training OFDM symbols, so the computational cost is $N_{\text {post }} \times J^{2}+O\left(J^{3}\right)$. Therefore, the computational cost to determine all the array weights is $N_{\text {post }} \times J \times O\left(M \log _{2} M\right)+M_{\text {sub }} \times\left(N_{\text {post }} \times J^{2}+O\left(J^{3}\right)\right)$. Besides this computational cost, from Fig. 2, it can be seen that the post-FFT array antenna requires $J$ FFT processors and $J \times M_{s u b}$ complex weight multiplications.

The pre-FFT type array antenna first needs to estimate the channel impulsre response at $J$ antenna elements. The channel impulsre response can be estimated by correlating the received signal with a part of the first half of the transmitted long training sequence with length of $M$ (see the second paragraph in Sect. 7 for the detail) within the period corresponding to the cyclic prefix $(L)$, so the computational cost is $J \times M \times L$. Secondly, it needs to calculate the correlation matrix $(J \times J)$ using $(L+M)$ samples in the second half of the long training sequence, so the cost is $(L+M) \times J^{2}$. Thirdly, it needs to solve the generalized eigenproblem given by (41) only once to determine all the array weights. The generalized eigenproblem can be solved with computational cost of $O\left(J^{3}\right)$ [15], so the computational cost to determine all the array weights is $J \times M \times L+(L+M) \times J^{2}+O\left(J^{3}\right)$. Besides this computational cost, from Fig. 3 , it can be seen that the pre-FFT array antenna requires $l$ FFT processor and $J$ complex weight multiplications. 
Table 1 Computational cost comparison.

\begin{tabular}{|c|c|c|c|}
\hline & Post-FFT & Pre-FFT & Eigenvector \\
\hline $\begin{array}{c}\text { Weight } \\
\text { Detimation and }\end{array}$ & $\begin{array}{c}N_{\text {post }} \times J \times O\left(M \log _{2} M\right) \\
+M_{\text {sub }} \times N_{\text {post }} \times J^{2} \\
+M_{\text {sub }} \times O\left(J^{3}\right)\end{array}$ & $\begin{array}{c}J \times M \times L \\
+M+L) \times J^{2} \\
+O\left(J^{3}\right)\end{array}$ & $\begin{array}{c}J \times M \times L \\
+(M+L) \times J^{2} \\
+O\left(J^{3}\right)\end{array}$ \\
\hline $\begin{array}{c}\text { Number of } \\
\text { FFT } \\
\begin{array}{c}\text { Processors } \\
\text { Number of } \\
\text { Complex } \\
\text { Weights }\end{array}\end{array}$ & $J$ & 1 & $K$ \\
\hline
\end{tabular}

On the other hand, the eigenvector combining array antenna also needs to solve the same eigenproblem only once to determine all the eigenvalues and all the corresponding initial array weights as the pre-FFT type array antenna. However, it furthermore needs to calculate the post multiplication weights given by (47) for $k=1, \cdots, K$ and $m=$ $1, \cdots, M_{\text {sub }}$. Taking into consideration that $\beta(k)_{1}, \cdots, \beta(k)_{M}$ can be jointly calculated through the $M$-point FFT operation for $k=1, \cdots, K$, the computational cost results in $K \times J \times O\left(M \log _{2} M\right)$. Therefore, the computational cost to determine all the array weights is $J \times M \times L+(L+M) \times$ $J^{2}+O\left(J^{3}\right)+K \times J \times O\left(M \log _{2} M\right)$. Besides this computational cost, from Fig. 4(a), it can be seen that the eigenvector combining array antenna requires $K$ FFT processors, and $K \times J+K \times M_{\text {sub }}$ complex weight multiplications.

Table 1 compares the computational cost among the post-FFT type, pre-FFT type and eigenvector combining array antennas. Taking into consideration of $N_{\text {post }}>10$ and $M, M_{\text {sub }} \gg J \geq K$, it is clear that the eigenvector combining is computationally less complex than the post-FFT type. Especially, if $K$ becomes smaller than $J$, the eigenvector combining becomes more advantageous over the post-FFT type.

Finally, it should be also noted that, even if the postFFT type uses an RLS algorithm with computational cost of $O\left(J^{2}\right)$ instead of the SMI algorithm, it has a larger computational cost than the eigenvector combining, because the computational cost is $M_{\text {sub }} \times O\left(J^{2}\right) \gg O\left(J^{3}\right)$.

\section{Numerical Results and Discussions}

Now, we evaluate the performance of the post-FFT type, pre-FFT type and eigenvector combining adaptive array antennas by computer simulation, whose block diagrams are shown in Figs. 2, 3 and 4(a), respectively. For computer simulation, the OFDM signal format in IEEE802.11a standard is assumed [16], namely, a coherent quadrature phase shift keying (QPSK) scheme is employed with a half-rate convolutional encoding/Viterbi decoding with a constraint length of 7 and a $12 \times 8$ block interleaver. One OFDM symbol is composed of 80 samples, where the cyclic prefix length is 16 samples and the useful symbol length is 64 samples. Furthermore, the OFDM symbol is generated with the 64point inverse FFT (IFFT), where only 48 subcarriers convey information, 4 subcarriers are known pilot signals and the other 12 subcarriers are virtual subcarriers.

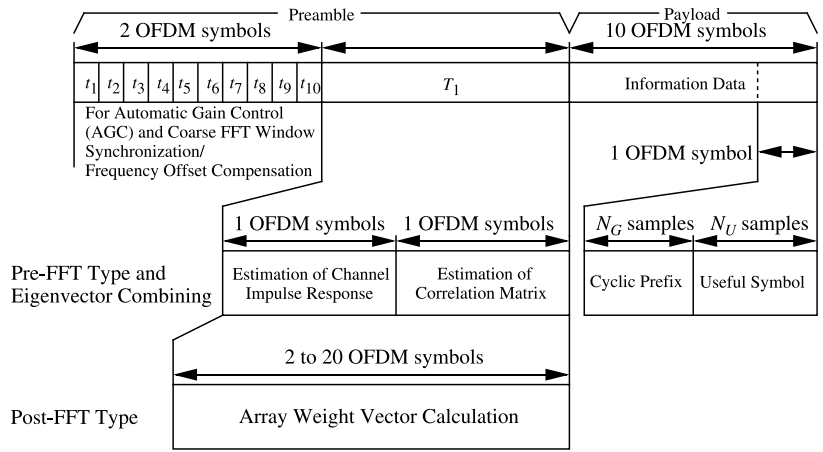

Fig. 5 Signal burst format.

The signal burst in IEEE802.11a standard is also assumed [16], namely, the one signal burst is composed of a preamble and a payload. Figure 5 shows the signal burst format. The length of the preamble is 4 OFDM symbols and that of the payload is set to 10 OFDM symbols. The first half of the preamble is composed of 10 repetitions of "a short training sequence" with length of 0.2 OFDM symbols $\left(t_{1}, \cdots, t_{10}\right)$ and is used for automatic gain control, FFT window timing synchronization and frequency offset compensation. In the following numerical demonstration, no frequency offset is assumed between the transmitter and receiver. The second half of the preamble is composed of a "long training sequence" with length of 2 OFDM symbols $\left(T_{1}\right)$. The first half of the long training sequence is used for estimation of the channel impulse response and the second half is used for estimation of the correlation matrix of the received signal. The long training sequence is long enough for the pre-FFT type and eigenvector combining array antennas, on the other hand, it is not long enough for the postFFT type array antenna because it requires a relatively long training sequence to solve the minimization problem given by (31). Therefore, for the post-FFT type array antenna, the length is extended up to 20 OFDM symbols.

Figure 6 shows an array geometry and a spatiotemporal channel model. An eight-element circular array with element spacing of half wavelength is assumed. In addition, three desired signals arriving within the cyclic prefix and one delayed signal beyond the cyclic prefix as cochannel interference satisfying (11) are assumed. We restrict our attention to an uplink transmission, where the position of a base station (BS) is sufficiently high so that few 
Envelope: Rayleigh distributed

Arrival Time: uniformly distributed within guard interval (Desired Signal) uniformly distributed within useful symbol (Interfering Signal)

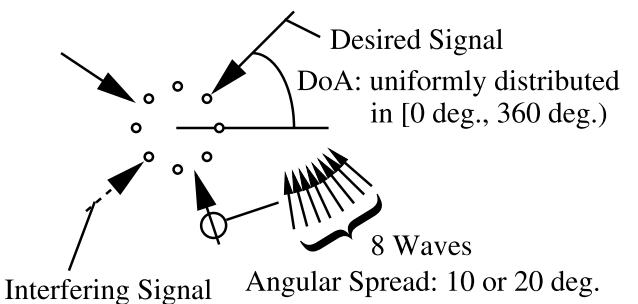

Fig. 6 An array geometry and a spatio-temporal channel model.

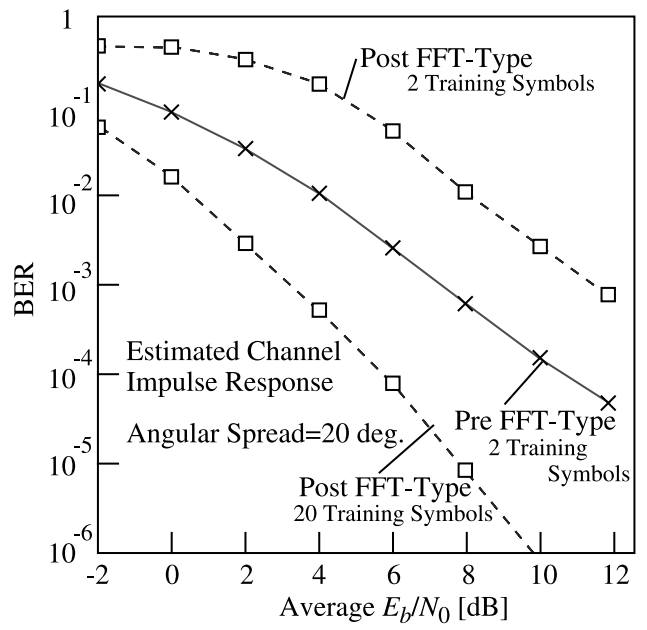

Fig. 7 BER of post-FFT and pre-FFT type array antennas.

local scatterings occur [18]. Therefore, each signal arrives forming a cluster with angular spread, which is composed of eight waves, the envelope is Rayleigh-distributed with the same power and the DoA is uniformly distributed in $[0$ degree, 360 degrees). The arrival time of the desired signal is uniformly distributed within the cyclic prefix, whereas that of the interfering signal is uniformly distributed within the useful symbol interval. Furthermore, the channel fading is slow enough so that the channel impulse response does not significantly change over one signal burst, namely, no Doppler frequency is taken into consideration within one signal burst.

Figure 7 shows the bit error rate (BER) versus the average ratio of the received energy per bit to the white noise power spectral density per antenna $\left(E_{b} / N_{0}\right)$ for the post-FFT and pre-FFT type array antennas, where the angular spread is set to 20 degrees and the SMI algorithm is used for both array antennas. For the post-FFT type array antenna, when setting the length of the training symbols to 2 , the BER is poor, whereas when setting the length of the training symbols to 20, the BER becomes excellent, but the burst efficiency is $0.33(=10 /(20+10))$. On the other hand, the preFFT type array antenna can achieve a much better BER, as compared with the post-FFT type array antenna with the

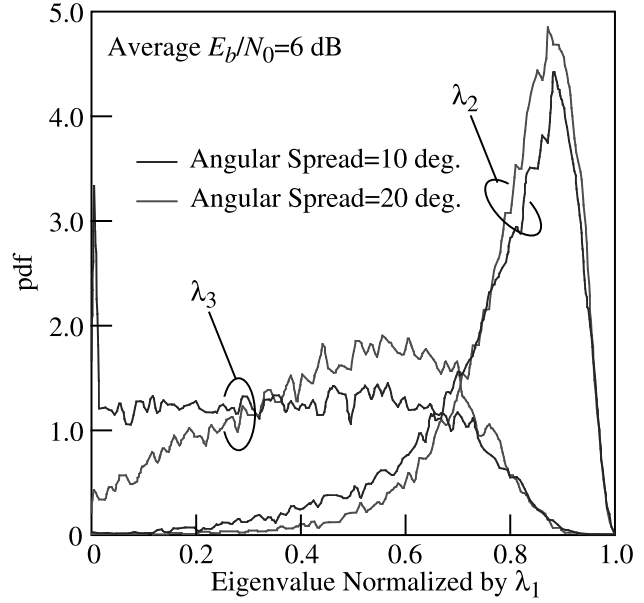

Fig. 8 Eigenvalue distribution.

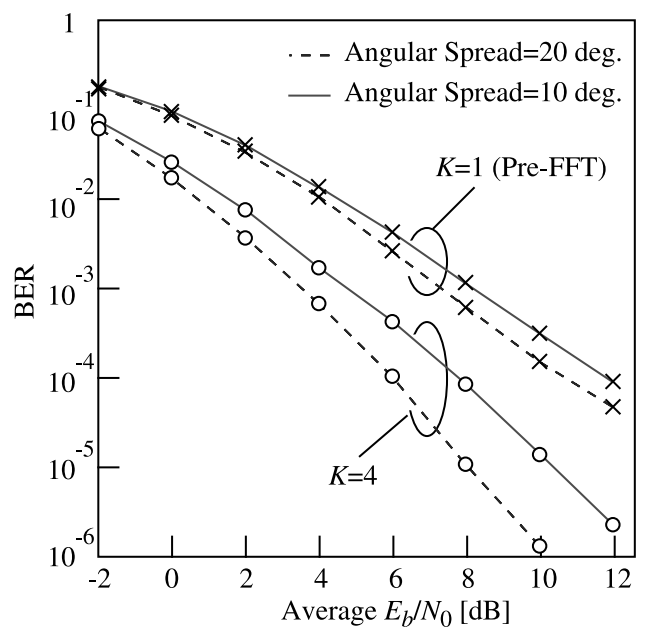

Fig. 9 BER of eigenvector combining array antenna (estimated channel impulse response).

same 2 OFDM symbol-long training symbols, whereas its performance is still inferior to that of the post-FFT type array antenna with the 20 OFDM symbol-long training symbols.

Figure 8 shows the simulated probability density function (pdf) of eigenvalues $\lambda_{2}$ and $\lambda_{3}$ normalized by the first largest eigenvalue $\lambda_{1}$. Here we assume that only a single Rayleigh-distributed signal with an angular spread arrives at the array antenna as shown in Fig. 6. In each simulation run, the DoA is randomly chosen, and the first, second and third largest eigenvalues are calculated by solving the (generalized) eigenproblem given by (41). Note that this is a case without interference, so the generalized eigenproblem becomes an eigenproblem. The distributions of $\lambda_{2}$ and $\lambda_{3}$ are obtained from the results of 20000 simulation runs, setting the average $E_{b} / N_{0}$ to $6 \mathrm{~dB}$. The occurrence of large $\lambda_{k}$ substantially increases when the angular spread increases (from 10 degrees to 20 degrees). This suggests that the BER performance of the eigenvector combining can become better as the angular spread increases. 


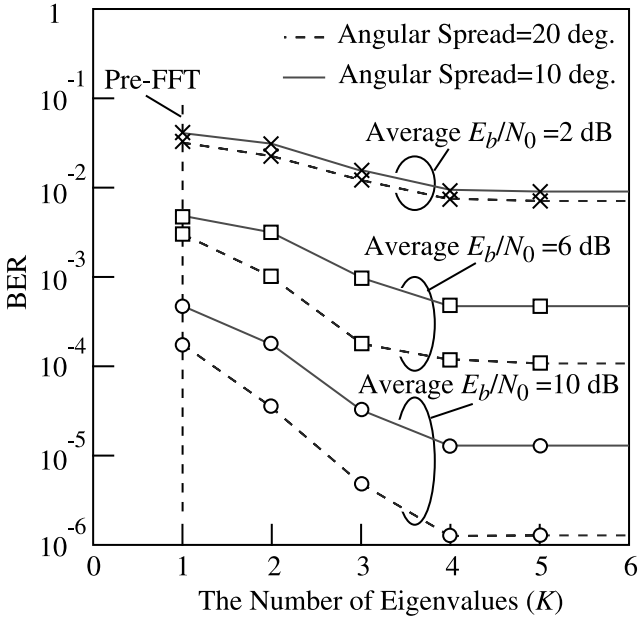

Fig. 10 BER performance versus $K$.

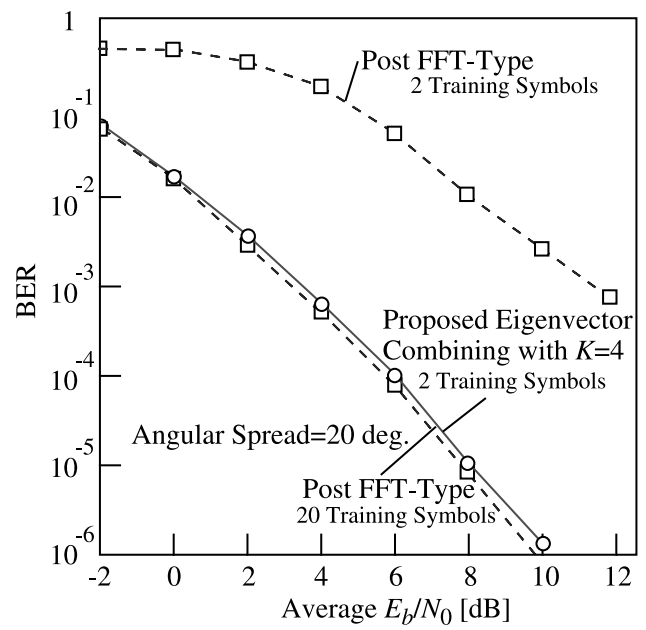

Fig. 11 BER comparison between eigenvector combining and post-FFT type.

Figure 9 shows the BER versus the average $E_{b} / N_{0}$ of the proposed eigenvector combining, where the channel impulse response is estimated with the first half of the long training sequence. The figure clearly shows that the BER becomes better for larger $K$. The BER also becomes better for larger angular spread, in the other words, the fading correlation among antenna elements decreases, as expected intuitively.

Figure 10 shows the BER versus $K$ for the eigenvector combining. The BER performance is improved as $K$ increases, i.e., more eigenvectors are employed for combining. We can calculate $8(=J)$ eigenvalues at most in this case, no performance improvement is observed even when $K$ is larger than 5. This implies that the signal is transmitted through 4 independent channels for the system parameter setting. The post-FFT type array antenna with 8 FFT processors is too much redundant.

Finally, Fig. 11 shows the BER comparison between the eigenvector combining and post-FFT type array anten- nas. When there is interference, it has not been completed to theoretically prove the equivalence between the two schemes, but the figure clearly shows that the performance of the eigenvector combining array antenna with $K=4$ and just 2 OFDM symbol-long training symbols is almost the same as that of the post-FFT array antenna with 20 OFDM symbol-long training symbols. Therefore, it is finally concluded that the eigenvector combining OFDM adaptive array antenna performs comparable to the post-FFT type array antenna while requiring less computational complex and shorter training symbols like the pre-FFT type array antenna.

\section{Conclusions}

This paper proposed a novel pre-FFT type OFDM adaptive array antenna called "Eigenvector Combining." The eigenvector combining is a realization of a post-FFT type OFDM adaptive array antenna through pre-FFT signal processing, and has the advantageous characteristics of the two types of array antennas, namely, excellent performance due to power efficiency inherent in post-FFT type and less computational complexity and requirement of shorter training symbols proper to pre-FFT type.

Numerical results showed that, for the case of three desired signals and one co-channel interfering signal, with the same 2 OFDM symbol-long as in the IEEE802.11a standard, the post-FFT type array antenna cannot work well at all, on the other hand, the proposed array antenna combining 4 eigenvectors shows excellent BER performance close to the lower bound.

The number of eigenvectors to be combined is a key parameter to determine the complexity of the proposed array antenna. System designers have to be careful about the system condition encountered, because different system conditions, such as the number of antenna elements employed and the wireless channel where the signal is transmitted, give different smallest number of eigenvectors obtaining a satisfactory performance.

\section{References}

[1] S. Hara and R. Prasad, Multicarrier Techniques for 4G Mobile Communications, Artech House Publishers, 2003.

[2] J. Chuang and N. Sollenberger, "Beyond 3G: Wideband wireless data access based on OFDM and dynamic packet assignment," IEEE Commun. Mag., vol.38, no.7, pp.78-87, July 2000.

[3] J.H. Winters, J. Salz, and R. Gitlin, "The impact of antenna diversity on the capacity of wireless communication systems," IEEE Trans. Commun., vol.42, no.4, pp.1740-1751, April 1994.

[4] R.T. Compton, Adaptive Antennas, Prentice-Hall, Englewood Cliffs, New Jersey, 1988.

[5] Y.(G.) Li and N.R. Sollenberger, "Adaptive antenna arrays for OFDM system with cochannel interference," IEEE Trans. Commun., vol.47, no.2, pp.217-229, Feb. 1999.

[6] S. Kapoor, D.J. Marchok, and Y.F. Huang, "Adaptive interference suppression in multiuser wireless OFDM systems using antenna arrays,” IEEE Trans. Signal Process., vol.47, no.12, pp.3381-3391, Dec. 1999.

[7] D. Bartolome and A.I. Perez-Neira, "MMSE techniques for space 
diversity receivers in OFDM-based wireless LANs," IEEE J. Sel. Areas Commun., vol.21, pp.151-160, Feb. 2003.

[8] M. Okada and S. Komaki, "Pre-DFT combining space diversity assisted COFDM," IEEE Trans. Veh. Technol., vol.50, no.2, pp.487496, March 2001.

[9] M. Budsabathon, Y. Hara, and S. Hara, "Optimum beamforming for pre-FFT OFDM adaptive antenna array," IEEE Trans. Veh. Technol., vol.53, no.4, pp.945-955, July 2004.

[10] S. Hara, M. Budsabathon, and Y. Hara, "A pre-FFT type OFDM adaptive array antenna with eigenvector combining," IEEE ICC2004, pp.2412-2416, Paris, France, June 2004.

[11] M. Budsabathon, Y. Hara, and S. Hara, "An OFDM adaptive array antenna with eigen-vector combining," IEICE Technical Report, AP-2002-157, Feb. 2003.

[12] D. Huang and K.B. Letaief, "Pre-FFT processing for coded OFDM systems with receive space diversity," Proc. IEEE WCNC'03, in CDROM, March 2003.

[13] M. Schwartz, W.R. Bennett, and S. Stein, Communication Systems and Techniques, pp.416-489, IEEE Press, Piscataway, NJ, 1996.

[14] S. Haykin, Adaptive Filter Theory, 3rd ed., Prentice-Hall, New Jersey, 1996.

[15] G.H. Golub and C.F. Van Loan, Matrix computations, 3rd ed., pp.375-390, Johns Hopkins University Press, Baltimore, MD, 1996

[16] IEEE Std. 802.11a, "Wireless medium access control (MAC) and physical layer (PHY) specifications: High-speed physical layer extension in the $5 \mathrm{GHz}$ band," IEEE, 1999.

[17] S.K. Mitra and J.F. Kaiser, Handbook for Digital Signal Processing, pp.548-553, John Wiley \& Sons, New York, NY, 1993.

[18] R.B. Ertel, P. Cardieri, K.W. Sowerby, T.S. Rappaport, and J.H Reed, "Overview of spatial channel models for antenna array communication systems," IEEE Pers. Commun., vol.5, no.1, pp.12-22, Feb. 1998.

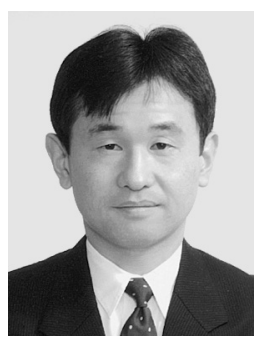

Shinsuke Hara received the B.Eng., M.Eng. and $\mathrm{Ph} . \mathrm{D}$. degrees in communications engineering from Osaka University, Osaka, Japan, in 1985, 1987 and 1990, respectively. From April 1990 to March 1997, he was an assistant professor in the Department of Communication Engineering, School of Engineering, Osaka University, and from October 1997 to September 2005, he was an associate professor in the Department of Electronic, Information and Energy Engineering, Graduate School of Engineering, Osaka University. Since October 2005, he has been a professor in the Department of Information Systems, Graduate School of Engineering, Osaka City University. In addition, from April 1995 to March 1996, he was a visiting scientist at Telecommunications and Traffic Control Systems Group, Delft University of Technology, Delft, The Netherlands. His research interests include wireless communications systems and digital signal processing.

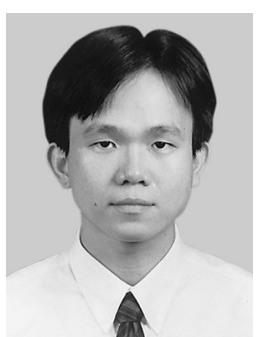

Quoc Tuan Tran received the B.Eng., and M.Eng. degrees in communications engineering from Osaka University in Osaka, Japan in 2003 , and 2005, respectively. He is currently pursuing the Ph.D. course at Osaka University, engaging in the research on digital signal processing techniques for wireless communications systems.

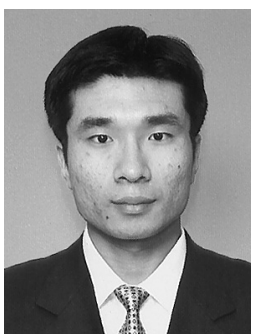

Yunjian Jia received the B.S. degree from Nankai University in Tianjin, China, in 1999 and the M.Eng. and Ph.D. degrees in communication engineering from Osaka University in Osaka, Japan, in 2003 and 2006, respectively. Since April 2006, he has been with the Central Research Laboratory, Hitachi Ltd., Tokyo, Japan. His research interests include mobile and indoor wireless communications systems.

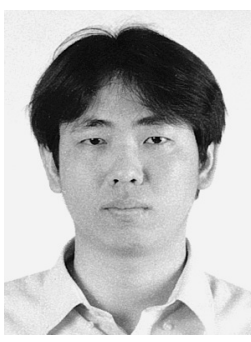

Montree Budsabathon was born in Bangkok, Thailand, on April 4, 1976. He received the B.Eng. degree (with first-class honors) from Chulalongkorn University, Bangkok, Thailand, in 1997 and the M.Eng. and Ph.D. degrees in communications engineering from Osaka University, Osaka, Japan, in 2000 and 2003, respectively. Now, he works for True corp., Thailand. His research interests include digital signal processing for wireless commutems and devices. nications systems and optical transmission sys-

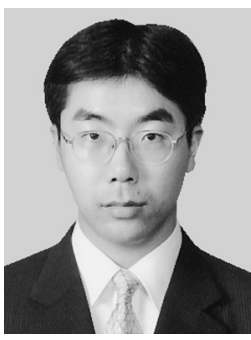

Yoshitaka Hara received the B.E., M.E. and Dr. Eng. degrees from the University of Tokyo, Tokyo, Japan, in 1993, 1995, and 2003, respectively. In 1996, he joined Mitsubishi Electric Corporation. From 1999 to 2001, he was also a senior research engineer at YRP Mobile Telecommunications Key Technology Research Laboratories Co., Ltd. Since 2003, he has been with the Mitsubishi Electric Information Technology Centre Europe B.V. (ITE). His research interests include wireless communication systems and adaptive antenna arrays. 